Case report

\title{
Pazopanib in clear cell sarcoma therapy
}

\section{Katarzyna Kryszczyszyn-Musialik ${ }^{1,2}$, Grzegorz Słomian ${ }^{1}$}

${ }^{1}$ Oncology Department with Hematological Subdivision,

Provincial Specialist Hospital No. 3 in Rybnik

Head of Department: Grzegorz Słomian

${ }^{2}$ Chair and Department of Environmental Medicine and Epidemiology,

Medical University of Silesia in Katowice

Correspondence: Katarzyna Kryszczyszyn-Musialik Oncology Department with Hematological Subdivision, Provincial Specialist Hospital

No. 3 in Rybnik, Poland 44-200 Rybnik, ul. Energetyków 46

tel.: +48 (32) 429-17-51

e-mail:kryszczyszyn@gmail.com

Received: 15.01.2018.

Accepted:

14.03.2018.

DOI: 10.24292/01.OR.140318 Copyright (C) Medical Education. All rights reserved.

\section{ABSTRACT}

Clear cell sarcoma (CCS) is a rare soft-tissue sarcoma and is characterized by a translocation $\mathrm{t}(12 ; 22)$ (q13;q12). Because of some similarities to maligned melanoma it is called melanoma of soft parts. CCS has characteristic clinical features. It is commonly reported in young adults as slowly growing tumor followed by aggressive course. Its features are: regional lymph node spread and tendency for local recurrence and a propensity for pulmonary metastasis. Metastatic CCS is a very aggressive disease with pure prognosis. Conventional chemotherapy has little role to play in the management of CCS. The article presents the case of a patient with metastatic CCS with massive lung dissemination, metastatic in liver, left adrenal, pelvis and superficial soft tissue. In patient pazopanib therapy resulted in nine-month control of the disease.

Key words: soft-tissue sarcoma, clear cell sarcoma, pazopanib 


\section{INTRODUCTION}

Clear cell sarcoma (CCS) is a rare mesenchymal cancer which is commonly reported in young adults [1, 2]. The estimated yearly incidence is 0,8 cases per 1 million people [2]. CCS is also called melanoma of soft parts because of many similarities to melanoma as histopathological view and clinical features - regional lymph node spread like melanoma, not typical route of metastasis for sarcomas - hematogenous spread and pure prognosis [1-5]. Microscopically CCS consists of rounded cells with clear or eosinophilic cytoplasm with large amounts of intracellular glycogen [4]. On immunohistochemical evaluation we can nearly always see expression S-100 protein, and also characteristic for melanoma such as HMB-45 and Melan-A. Only molecular examination allows to clearly distinguish CCS from melanoma $[4,6]$. Clear cell sarcoma is characterized by a translocation $\mathrm{t}(12 ; 22)$ (q13;q12) and fusion of EWS (22q12) (Ewing sarcoma gene) with ATF1 genes (12q13) (activating transcription factor 1) [1, 2, 4, 6]. That typical translocation has not been reported in melanoma. That also has clinical implication. The activation of ATF1 gene affects cell viability. In future it can be the goal of targeted treatment [2].

CCS involves the peripheral tendons and aponeuroses. It often spreads to regional lymph node $[1-5,7,8]$. Radical treatment consists of radical resection, sentinel lymph node biopsy (SLNB) and postoperative radiotherapy [8]. The role of adjuvant chemotherapy has not been confirmed [4]. This disease has bad prognosis. CCS can disseminate even after many years, and it is resistant for conventional chemotherapy. The targeted treatment can be a potential therapy option $[1,2,4]$.

Pazopanib is a synthetic indazolpyrimidine. It is a multitargeted tyrosine kinase inhibitor with activity against vascular endothelial growth factors 1, 2, and 3, and platelet-derived growth factors $[9,10]$. It has been registered to treat patients with non-adipocytic soft-tissue sarcoma (STS) after failure of standard chemotherapy or with progression in 12 month after neoadjuvant or adjuvant therapy [9]. PALETTE trial - a randomized, double-blind, placebo-controlled phase III trial proves that pazopanib has single-agent activity in patients with advanced non-adipocytic soft-tissue sarcoma. The most common histological types of soft-tissue sarcoma were allowed; excluding all types of adipocytic sarcoma, embryonal rhabdomyosarcoma, chondrosarcoma, osteosarcoma, Ewing tumors, primitive neuroectodermal tumor, gastrointestinal stromal tumor, dermatofibrosarcoma protuberans, inflammatory myofibroblastic sarcoma, malignant mesothelioma and mixed mesodermal tumors of the uterus. The patients received either pazopanib $800 \mathrm{mg}$ once daily or placebo with no subsequent cross-over. Patients were included in that trial after progressing despite previous standard chemotherapy. More than 300 patients were registered. Median progression-free survival (PFS) was 4.6 months for pazopanib compared with 1.6 months for placebo. The most common adverse events were fatigue (65\% in pazopanib group and $49 \%$ in placebo group), diarrhea (pazopanib 58\% vs placebo $16 \%$ ), nausea (pazopanib 54\% vs placebo $28 \%$ ), weight loss (pazopanib $48 \%$ vs placebo $20 \%$ ) and hypertension (pazopanib $41 \%$ vs placebo 7\%) [9].

The article presents the case of a patient with metastatic CCS with massive lung dissemination, metastatic in liver, left adrenal, pelvis and superficial soft tissue. In this case pazopanib therapy results in nine-month control of disease -36 week progression-free survival.

\section{CASE STUDY}

In June 2014 42-year-old woman was admitted to the Oncology Department after failure of standard chemotherapy because of advanced clear cell sarcoma. She had the history of treatment in the nearby hospital. The treatment of the patient was as follows.

In January 2013 histopathological confirmation has been obtained after inguinal lymphadenectomy. The histopathological examination was: "Clear cell sarcoma IM15/10 HPF. Neoplasm with high malignancy potential. EWSR rearrangement present in $13 \%$ interphase nucleus". During oncological consultation in the nearby hospital positron emission tomography (PET) was recommended (May 2013). PET suggested acral localization of primary tumor (right sole). The patient admitted she had a change resected from her right sole in the past. There is no medical documentation about it available. The patient was qualified to a radicalizing operation - radical right inguinal lymphadenectomy (June 2013). In January 2014 the patient received chemotherapy CYVADIC (cyclophosphamide, vincristine, doxorubicin, and dacarbazine) because of the advanced disease. After the fifth course, the chemotherapy was ended because of progression disease (PD).

The patient was directed to the next treatment.

During qualification to pazopanib computer tomography (CT) on chest, abdomen and pelvis with contrast showed huge progression of the disease. Massive metastases were presented in lung, in liver, left adrenal, pelvis, in intraperitoneal lymph nodes and in paraspinal soft tissue, on level L1/L2 they had 
penetrated into the spinal canal. Many soft tissue metastasis were examined in subcutaneous tissue. Maximal subcutaneous, red tumor was $45 \times 50 \mathrm{~mm}$ and it was located on the left side of the neck.

Despite the advanced disease the clinical condition of the patient before the treatment was scored 0 according to ECOG (Eastern Cooperative Oncology Group). There was no chronic disease in medical history. In echocardiography left ventricular ejection fraction (LVEF) was $64 \%$. The disease range was assessed in chest, abdomen and pelvis $\mathrm{CT}$, neck ultrasonography and physical examination. In the lab test all the parameters were correct.

\section{Treatment}

The patient was included in pazopanib in soft tissue sarcoma therapy. She received pazopanib $800 \mathrm{mg}$ once daily (one cycle - 28 days). During the second cycle patient has presented hypertension. Ramipril $2.5 \mathrm{mg}$ once daily was recommended. During that therapy blood tension was proper. Tolerance of the pazopanib was good. Other adverse events were metallic taste in the mouth and hair depigmentation. In the lab test elevation of transaminases appeared in grade G1 (according CTCAE Common Terminology Criteria for Adverse Event) and it failed away. After 3 months therapy metastases shrank - in RECIST 1.1 (Response Evaluation Criteria In Solid Tumors 1.1) it was partial response (PR). After next 3 months stabilization was noticed - SD (stable disease). After the $9^{\text {th }}$ cycle there was progression $-58 \%$. The treatment was ended.

Pazopanib was administered for 36 weeks. In that period the patient had good disease control with negligible adverse events, which ware acceptable. After the treatment rapid progression of the disease was observed. After next 3 month massive lymph edema has appeared on right leg. It was consequence of the enlarged subcutaneous tumors in the right inguinal. In subcutaneous tissue all the tumors grew and many new lesions on neck, head and back appeared. In the blood examination anemia occurred. The CT scan showed massive multiorgan progression. There was an attempt of monotherapy with ifosfamide (dose $2.4 \mathrm{~g} / \mathrm{kg}$, day $1^{\text {st }}-3^{\text {rd }}$ ) The patient died after the first cycle of the chemotherapy.

\section{DISCUSSION}

Clear cell sarcoma is a rare, characterized by great malignancy and bad prognosis neoplasm [1-5, 7], with typical regional lymphatic vessels spread $[1,2,8]$. Conventional chemotherapy has minimal activity in CCS. It is often resistant to standard chemotherapy and radiotherapy $[3-5,7,11-13]$

Jones et al. presented the results of the series of patients with CCS receiving chemotherapy. In the study database from Royal Marsden Hospital and Memorial Sloan-Kettering Cancer Center with CCS was analyzed. 24 patients were treated with palliative first-line systemic therapy. The median progression-free survival following palliative first-line chemotherapy was 11 weeks. 12 patients were treated with second-line systemic therapy, 11 of them progressed and 1 achieved stable disease. One patient had stabile disease following fourth-line chemotherapy, which was maintained for 4 months. The response was assessed every 2 cycles of the treatment. Investigators used various chemotherapy regimen: anthracycline, ifosfamide, platinum, vinblastine, vincristine, thalidomide and others. The median overall survival from commencing chemotherapy was 39 weeks with response rate (RR) of 4\% [4]. According to this and other analysis conventional chemotherapy has minimal activity in clear cell sarcoma $[3-5,7,11-13]$.

In CCS similar with alveolar soft part sarcoma and translocation-associated renal cell cancer is a MIT (microphthalmia transcription factor) associated malignancy. In CCS translocation $\mathrm{t}(12 ; 22)(q 13 ; q 12) /$ EWSR1-ATF1 fusion activates MIT leading to overexpression of MET. MET is the receptor for hepatocyte growth factor (HGF), which is responsible for cell survival, adhesin, invasion, migration and angiogenesis. MET inhibitors can be potential treatment for this translocation-related neoplasm [4]. In the results of the phase II trial for tivantinib (ARQ197) published by Goldberg in 2012, the OS for the CCS cohort was 5 months [14]. HDAC (histone deacetylase inhibitors) and fibroblast growth factor receptor 1 (FGFR1) are currently being investigated in CCS therapy $[15,16]$.

The investigation of clear cell sarcoma cell line (Hewga-CCS) confirmed the antitumor effects of pazopanib on Hewga-CCS. These cells were incubated for 24 to $72 \mathrm{~h}$ with pazopanib at concentrations of $0-20 \mu \mathrm{mol} / \mathrm{l}$, followed by counting the number of living cells. These data indicated that pazopanib has a direct antiproliferative effect on Hewga-CCS cells in vitro. The c-MET pathway is a potential target for pazopanib in Hewga-CCS cells [17].

\section{CONCLUSIONS}

The article presents the case of a patient with metastatic CCS pazopanib therapy resulting in long control of the disease. Dur- 
ing therapy the state of the patient was good and pazopanib was well tolerated. It was essential to monitor potential toxicity like hypertension, increased concentrations of liver enzyme. Adequate control of that could prolong treatment by avoiding serious complications (G3-G4 according to CTCAE) [18].

In this case study chemotherapy has no activity. After pazopanib therapy rapid progression of the disease, deterioration of the general condition of patient and death after the first cycle of ifosfamide treatment were observed.

In palliative CCS therapy targeted treatment is a chance for improvements in progression-free and overall survival for patients with this rare condition. New investigation and clinical studies of novel systemic agents will hopefully lead to the evolution of treatment of this poor prognosed sarcoma.

\section{References}

1. Rutkowski P, Nowecki Zl. Mięsaki tkanek miękkich. Medical Tribune Polska, Warszawa 2009: 247-249.

2. Jeziorski A. Biblioteka chirurga onkologa. Tom 3. Mięsaki tkanek miękkich. Rutkowski P. (ed). Via Medica, Gdańsk 2015: $217-221$.

3. Sara AS, Evans HI, Benjamin RS. Malignant melanoma of soft parts (clear cell sarcoma): study of 30 cases. Cancer 1990; 65: 367-374.

4. Jones RL, Constantinidou A, Thway K et al. Chemotherapy in clear cell sarcoma. Med Oncol 2011; 28: 859-863.

5. Deenik W, Mooi WJ, Rutgers EJ et al. Clear cell sarcoma (malignant melanoma) of soft parts: clinicopathologic study of 30 cases. Cancer $1999 ; 86$ : 969-975.

6. Hisaoka M, Ishida T, Kuo TT et al. Clear cell sarcoma of soft tissue: a clinicopathologic, immunohistochemical and molecular analysis of 33 cases. Am J Surg Pathol 2008; 32: 452-460.

7. Kawai A, Hosono A, Nakayama R et al. Clear cell sarcoma of tendons and aponeuroses: a study of 75 patients. Cancer 2007; 109: $109-116$.

8. van Akkoi AC, Verhoef C, van Geel AN et al. Sentinel node biopsy for clear cell sarcoma. Eur J Surg Oncol 2006; 32: 996-999.

9. van der Graaf WT, Blay JY, Chawla SP et al. Pazopanib for metastatic soft-tissue sarcoma (PALETTE): a randomised, double-blind, placebo-controlled phase 3 trial. Lancet 2012; 379: 1879-1886.

10. Kumar R, Knick VB, Rudolph SK et al. Pharmacokinetic-pharmacodynamic correlation from mouse to human with pazopanib, a multikinase angiogenesis inhibitor with potent antitumor and antiangiogenic activity. Mol Cancer Ther 2007; 6: 2012-2021.

11. Kuiper DR, Hoekstra HJ, Vet RP et al. The management of clear cell sarcoma. EJSO 2003; 29: 568-570.

12. Mackey SL, Hebe J, Cobb MW. Melanoma of the soft parts (clear cell sarcoma): a case report and review of the literature. J Am Acad Dermatol 1998; 38: 815-819.

13. Clark MA, Johnson MB, Thway K et al. Clear cell sarcoma (melanoma of soft parts): The Royal Marsden Hospital experience. Eur J Surg Oncol 2008; 34(7): 800-804.

14. Wagner AJ, Goldberg JM, Dubois SG et al. Tivantinib (ARQ 197), a selective inhibitor of MET, in patients with microphthalmia transcription factor-associated tumors: results of a multicenter phase 2 trial. Cancer 2012; 118: 5894-5902.

15. Liu S, Cheng H, Kwan W et al. Histone deacetylase inhibitors induce growth arrest, apoptosis and differentiation in clear cell sarcoma models. Mol Cancer Ther 2008; 7: 1751-1761.

16. Yokoyama S, Feige E, Poling LL et al. Pharmacologic suppression of MITE expression via HADAC inhibitors in the melanocyte lineage. Pigment Cell Melanoma Res 2008; 21: 457-463.

17. Outani $\mathrm{H}$, Tanaka T, Wakamatsu T et al. Establishment of a novel clear cell sarcoma cell line (Hewga-CCS), and investigation of the antitumor effects of pazopanib on Hewga-CCS. BMC Cancer 2014; 14: 455.

18. Domagała-Haduch $M$, Jasiówka M, Nowak $Ł$ et al. Complications of palliative antiangiogenic therapy in patients with colorectal cancer. OncoReview 2016; 6: 199-204.

Authors' contributions: Katarzyna Kryszczyszyn-Musialik: 85\%; Grzegorz Słomian: 15\%

Conflict of interests:

Financial support:

None.

Ethics:

The paper complies with the Helsinki Declaration, EU Directives and harmonized requirements for biomedical journals. 